\title{
The Rhetoric of Science: Authority and Duty in an Article from the Exact Sciences
}

\author{
Bruno Latour \\ École des Mines de Paris \\ Paolo Fabbri \\ Bologna University \\ Translated by \\ Sarah Cummins \\ Université Laval
}

This paper, an important translation of a 1981 article published in French*, is one of the earliest critical close readings of a scientific text, and one of the most revealing. The authors blend a continental literary-critical sense of rhetoric with a British epistemological sociology to examine a significant but typical scientific article. The analysis is noteworthy for its revelation of the deeply textual nature of science, for its treatment of authority as the right to assert, for its introduction of the notion of modalities with respect to scientific assertions (a concept that Latour and Woolgar's Laboratory Life would bring to wide currency), and for the general way in which it opens up scientific discourse to critical analysis. Also noteworthy is the brief postscript assembled by the original publishers to give voice to the umbrage the scientific authors took over Latour and Fabbri's analysis.

It is often said that science is "the set of true statements" (Wittgenstein) and that scientific style is characterized by impersonal statements such as "Substance A acts on substance B." To define scientific style this way is to confuse science as it is presented in textbooks with the kind of scientific writing exchanged within the field. Rather than analysing scientific style in its popularized form, we prefer instead to go through, and lead readers through, an article situated at the "frontier" of a particular science. Our aim is not to propose a theory of scientific style but to encourage phi- 
losophers and sociologists to analyse scientific texts as they are actually written. We have selected an article in neuroendocrinology, which appeared in 1962 in the Comptes rendus of the Paris Academy of Science**. This article was ideal for our purposes because it constitutes the main production of a laboratory whose primary product is articles (a group of ten Ph.D.'s produces 40 articles per year on average). One "popularizing" article is produced for forty "pioneering" articles. In addition to articles, the laboratory also produces informal communications between researchers, researchers and technicians trained in the laboratory, and substances which are sent to other researchers for study.

The analysis presented here is a cross between a study in the sociology of science and a semiological study. The sociology of science, particularly in the Englishspeaking world, has long used articles as the basic unit for calculating researchers' productivity and for defining the limits and measuring the growth of various disciplines.'

Publications of the Institute for Scientific Information in Philadelphia provide material for numerous studies in the sociology of science, in particular the Science Citation Index, which lists annually the number of citations each article has received. A similar index exists for the social sciences. The review Current Contents publishes a weekly summary of this literature.

This diagram presents the four most common approaches to scientific literature. For practical reasons, which will be explained elsewhere, scientific knowledge can be considered as a network of articles that influence one another through the intermediary of scientists (column C). But it is also possible (as shown in column B) to consider that scientists influence one another through the intermediary of articles, thereby obtaining recognition. In this view, articles engender other articles and researchers exchange only prestige. These are the two paths taken by the sociology of science. They differ from the usual analysis that sees Science as being made up of either Scientists (when there are only authors) or Knowledge (when there are only networks of articles). Columns A and D summarize these two ways in which scientific activity is subsumed either in individuals or in productions. In this article, our approach is of course different from the two common analyses, but also differs from the two paths taken up until now by the sociology of science, because we consider both the strategies of authors and the interactions among texts (the zigzag line in the diagram). 
Hagiographic images and sociographic images of science

canonization


In all of these studies only citations are considered, never the content of the articles and certainly not their style. ${ }^{2}$ Semiotic analysis, for its part, does indeed study the forms of texts but the methods developed ${ }^{3}$ are not applied to texts of natural sciences, although some attempts have been made to apply them to discourse in the humanities. ${ }^{4}$ We were thus interested in using modern literary analysis to bring the sociology of science into the heart of scientific articles, and to thereby determine whether the literature of the exact sciences obeys general rules valid for all forms of literature.

\section{Sociological markers}

The article we present here, like all others, shows the signs of various elements whose influence the sociologist attempts to weigh. The text contains six markers that explicitly refer to the conditions under which it was produced. The keyword "ENDOCRINOLOGIE" / endocrinology allows the article to be slotted into various classification systems. However, the article belongs properly to neuroendocrinology, a 30year-old discipline born of a cross between two others, which is not yet sufficiently well-established to have its own index in the Comptes rendus, although it has its own journals, university chairs, and conferences. In the text, the word "endocrinologie" marks the history through which disciplines are opened, become institutionalised, and establish their borders. ${ }^{5}$ The order of presentation of the four authors reflects the power relationships within the group; these relationships, of great interest to American sociologists, are a function of the microsociology of the research team. ${ }^{6}$

An asterisk refers the reader to the date " 23 juillet 1962" / July 23, 1962, the date of the Académie des sciences session where the paper was presented.7 This date, which is different from the publication date, allows for the settlement of disputes about priority. Since at the frontier of a science there can exist no undated idea, the time lag between the date of reception and the date of publication of an article is an important factor when choosing a journal. The Comptes rendus are known for publishing within a few weeks. The "Note" — dated, titled, assigned, and indexed — is in addition "présenté par M. Robert Courrier" / presented by Mr. Robert Courrier. The Note had to go through this member of the academy in order to be read before the authors' peers. Mr. Courrier is among those - known in English as "gatekeepers" - who decide whether or not certain authors appear. Their power is often considerable. On page 5 of the article there are two other markers, referring respectively to the research fund and to the institution that made the research possible. The amount of money is not mentioned, but the grant number is. This number represents a complicated system under which the research group's proposal was evaluated and accepted by a group 
of peers, in this particular case both by officials in the U.S. department of health and, in France, by the Centre national de la recherche scientifique (national scientific research centre). The Collège de France is the institution that agreed to provide physical and administrative facilities to the group. But the space granted is a socialized space, which is in addition a rare commodity. Around this space there always forms a web of calculation and sometimes intrigue: Why did this researcher get so much space? Why is there access to this instrument? Why is this administrative facility provided?

Thus, even before reading the article, the sociologist of science finds a very rich object that makes explicit reference to a twin set of strategies: those of the author (selection of the discipline, the title, the timing, the journal) and those of institutions (selection of the gatekeeper, the funders, the laboratory). The article also implicitly refers to a whole set of conflicts that do not appear as such in the text. The production of this piece of knowledge stands at the intersection of all of these practices. If any one of the conditions were to vary, the article we have before us would be different.

\section{Rhetoric and communication}

In going through the text, we will be using several terms that are not yet in common usage; we define them briefly here. A distinction must be made between the "pragmatic" dimension of the text - by whom, for whom, and for what reasons it is written - and its strictly textual dimension. But within the text itself, a distinction must be made between the system of "enunciation" (énonciation), whereby the author chooses whether or not to introduce the pragmatic dimension, and the system of "assertions" (énoncés), which makes no reference to the author. In the following table, we list all the markers with which the author makes his own presence felt in the text. It can be seen that, contrary to what is commonly assumed, this text, while scientific, is not in the least impersonal. Among the assertions (énoncés) themselves, a distinction must be made between propositions and "modalizations" - that is, whatever may modify a proposition. Last, the term "anaphor" designates any reference to the text itself or to other texts.

The most striking feature of this text is obvious from skimming through it briefly: it is riddled with references. On page 5 of the Note are listed ten references that provide the context of the article. The first seven of these are previously published writings that the Note will discuss, and the last three are descriptions of methods that are referred to by a number: "préparé comme dans (8)" / prepared as in (8), or "adaptée de la méthode de McKenzie (9)" / adapted from McKenzie's method (9). All of these references are to works outside the text, but other references operate within the text. For example, the whole of paragraph 5 is a reference to the table on page 3 of the text. The table itself refers to the figure on page 4 . The table and the figure were produced 


\section{Enunciation and assertion (Énonciation et énoncés)}

enunciation (enonciation)

(The first number refers to the page number of the article reproduced here, and the second to the line number.)

\section{Ist person}

nous montrons / we show 1, 6

nous appellerons / we shall call 1, 8

l'un de nous / one of us 1, 13 ses / his 1, 14

notre point de vue / our point of view I, 16

nous utilisons / we use 1, 32

nous utilisons / we use 2,2

nous avons montré / we have shown 2, 18

nous parlerons donc/ we shall thus speak 2, 43

\section{3rd person}

plusieurs auteurs / several authors 1,12

Reichlin 1, 15

Les résultats de Schreiber/Schreiber's results 1,18

on prèlève / literally, one takes 1,29

on a décrit / literally, one has described, 2, 37

\section{modalisations}

postuler / postulate 1,2

qui serait / which supposedly is 1,3

ont déjà dit / have already claimed 1, 12

exprimé ses réserves / expressed reservations 1, 14

les mèmes réserves / the same reservations 1,15

n'ont pas été réunies les conditions / the conditions were not assembled 1, 19

sans préjuger / without prejudging 2, 44

correspond certainement / definitely corresponds 5, 1

probablement / probably 2,13

\section{assertions (énoncés)}

\section{active}

de nombreux résultats / many findings 1,1

une neurohumeur qui serait / a neurosecretion which is

apparently 1,3

une substance qui répond / a substance meeting 1,7

la méthode a conduit / the method led 1, 10

les réserves doivent s'appliquer / reservations must apply

l, 14

la fraction agit / the fraction acts $\mathbf{1}, 20$

la filtration se fait / filtration takes place 1, 28

la zone correspond / the zone corresponds 2,11

l'activite persiste / the activity continues 2,13

elle correspond / it corresponds 2, 13

elle correspond / it corresponds 2, 15

fraction n'est plus active / fraction is no longer active 2,18

la réponse est identique / the response is identical 2, 19

l'activité dépend / the activity depends 2, 20

elle est stable / it is stable 2, 21

l'hydrolyse ne détruit pas / hydrolysis does not destroy 2, 23

l'injection donne / the injection results in 2, 25

la fraction B ne produit pas / fraction B does not produce

2,27

les doses n'ont pas donné / the doses did not produce 2, 34

la fraction B n'a pas / fraction B does not have 2, 36

les activités correspondent / the activities correspond 2, 42

la substance correspond / the substance corresponds 5,1

\section{passive}

les résultats rapportés par / results reported by 1,18 un extrait préparé / an extract prepared 1,25

un extrait est repris / an extract is recovered 1, 26

un extrait est appliqué / an extract is applied 1,27

une expérience est réalisée / an experiment is carried out

1,30

souris soumise / mouse subjected 1, 34

les détails seront rapportés / the details will be reported 2, 4

la vasopressine est mesurée / vasopressin is measured 2, 5

deux zones sont retrouvées / two zones are again found 2, 29

la fraction a étét retrouvée / the fraction was again found

2,29

l'activité est localisée / activity is located 2, 32

aucun effet n'a été trouvé / no effect was found 2, 38 
in the laboratory using laboratory instruments. They are, in a sense, the symbolic writings of these instruments, and they make up the infratext upon which the demonstration will be built. By a third type of anaphor, paragraphs 1,2 and 6- which we will designate henceforth as "text A" - refer to paragraphs 3, 4, and 5 ("text B"). Thus, expressions such as "par cette Note nous montrons" / in this Note we show (page 1, line $5)$ and "les activités decrites plus haut" / the activities described above $(2,40)$ show that text $\mathrm{A}$, which itself operates on the context, is established in reference to text $\mathrm{B}$, which in turn is authorised by the infratext. The writings of the instruments make a transformation of the literature possible. Lastly, a fourth type of anaphor: the text itself is referenced in its entirety by the title and usually (although not in this case) by an abstract. The title or the abstract do not simply indicate or evoke the text, but are a concentration of all its information, a rare occurrence in the humanities and an impossibility in literature. The proliferation of references modifies the classic problem of the referent (by referent we mean simply that of which a given discourse speaks). In other literary practices, there is either a referent which is not textual or else there is no referent at all - as in fiction intended as such. In the article presented here, there is indeed a referent, but it consists of an accumulation of texts: the context upon which the article acts, the infratext upon which it is based, part $B$, upon which part $A$ is based. It is as if the paper's solidity - some might say its objectivity — stems from the correspondences established by interleaving each of these different layers of texts. Beneath the scientific text one finds not nature, but the literature of instruments.

\section{The problem of the information transmitted}

"Upstream" or "downstream" from the text we find not nature (the ultimate referent) but other texts that cite this one or that it cites. Other forms of writing either focus attention on a unique, irreplaceable object (this is the case with a novel or a poem) or transmit information (as with a textbook, a directory, etc.). This article, however, is by definition replaceable, because one must be able to build upon it. If this article is true, another article may refer to it in a half-sentence; if it is false, other articles may challenge it or it may be totally ignored. ${ }^{8}$ The article is not like a novel, but it is not like a telephone directory either. In fact, leaving aside paragraph 3 , the amount of information transmitted by the article is quite small. The set of technical words - "diencéphale" / diencephalous, "TRF", "acétate de pyridinum" / pyridinum acetate - should not be construed as information, for these words designate precisely that which, for the small group of peers for whom the text is intended, is not called into question. This jargon may make the text obscure to uninformed readers but offers no new information to scientists. 
What, then, is the new information transmitted by this text which would justify its five pages? The text does not transmit information; instead it acts. For five pages it seeks to convince. Convince of what? That an important shift, an important operation has been achieved in the literature. Convince whom? The group of peers, by definition the most difficult group to convince, for it is made up of people who are challenged by this achievement, this "victory."

How will the text seek to convince? By means of other texts produced in the laboratory and "produced" here as evidence. Through analysis of this "victory" - the term is used here as in politics, sports, or games - we are able to penetrate the content of the article. The operation performed by the article is summarised in the title and explained in paragraph 1. In the first sentence, the speaker (i.e. the author or authors) is replaced by an impersonal ("de nombreux résultats" / numerous findings) which "ont conduit" / have led to the modalization of a proposition. The modalization is marked twice: first by the verb "postuler" / postulate and second by the use of the conditional ("qui serait / which is taken to be, which apparently is; literally, which would be). The proposition could be translated into ordinary language: there exists in the hypothalamus a substance that controls secretion by the pituitary gland of the hormone TSH, which in turn regulates secretion of hormones by the thyroid. ${ }^{9}$ The action the text performs is seen in a twin transformation in the second sentence. Instead of an impersonal construction ("de nombreux résultats") the speaker appears: "nous montrons" / we show; instead of modalization ("postuler l'existence d'une substance qui serait" / postulate the existence of a substance which is taken to be) there is an affirmation: "l'existence d'une substance qui correspond aux caractéristiques attendues / the existence of a substance that has the expected characteristics. Through this twin transformation, a possibility under discussion takes on an indisputable existence. To understand this shift, it is necessary to grasp what is at stake. Over the previous 20 years a paradigm had been constructed, on the basis of physiological data, whereby the endocrine system, far from regulating itself (feedback) could be controlled by the nervous system via the hypothalamus. In the 60 s this paradigm was not challenged. But since the implicit ontology of endocrinology is resolutely substantialist, a subparadigm had to be developed, postulating (as in Aristotle) the existence of homogeneous, specific, discrete substances. Until this article was published, neuroendocrinology had offered a chaos of findings, claims, evidence, and counter evidence, but no clearly identified substance. In contrast, this article - or more precisely, the group of articles surrounding it - constructs a clearly defined object; that is at least what it says it does and what it is recognised by other texts that cite it. Every text seeks to convince, but the scientific text achieves this effect by developing layers of texts consistent with one another which serve as mutual referents. Here, for example, bioassays, 
the stages of purification, the rules of the procedure followed, the statistical analysis - all are in agreement. This agreement is a break from the disagreements that preceded it. Where before there were claims, now there exists an object. In order to grasp the importance of this shift, it must be understood that it took more than eight years, close to $\mathbf{1 0 0}$ articles, several million dollars, and a half-dozen people to move from this 1962 article, which demonstrates the existence of the substance, to a 1969 article in which its chemical formula is demonstrated (Comptes rendus, Académie des sciences, 1969 , vol 269, December 9, 1969). The 1962 text reviews the paradigm, concentrates it into three words ("de nombreux résultats" / numerous findings) and one reference ("voir la récente revue de Bogdanove [1]" / see the recent review by Bogdanove [1], p. 1, l.5). This action makes the preceding field of argumentation obsolete. A new field is opened which can be called "isolation and characterisation of TRF." When the chemical formula of this substance was established in 1969, all of the literature produced in the intervening eight years in turn became obsolete. All subsequent studies took this final paper as point of departure and opened new fields based on it. The field of discussion can be defined in strictly intertextual terms - that is, by a hundred or so papers that explicitly refer to one another and which are all subsumed in the latest one.

\section{Detour via polemics}

The position that the author wishes to take is already occupied. "Plusieurs auteurs ont déjà dit avoir mis en évidence et purifié la substance TRF" / Several authors have already claimed to have identified and purified the substance TRF, p. 1, 1. 12). It will thus be necessary to dislodge these authors from their position, which commands the opening of the field of study. Battle is joined via a stylistic device ("ont dit avoir montré" / claim to have shown), which stands of course in contrast with "nous montrons" / we show in line 6 . The same operation could have been performed more brutally or more delicately. In an American article there would have been a polite remark to the effect that findings of the two laboratories do not match up. At this level, professional and national customs, individual education, and the art of writing may intervene to modulate writing operations largely beyond the author's control. Our study of operations cannot be reduced to a matter of individual style. It supports neither those who claim that scientific articles "have no style," nor those who find that scientific articles display only an individual style. To adopt either one of these positions is to fail to distinguish among the writing strategies imposed by the state of knowledge attained, rhetoric imposed by the scientific community, and minor stylistic devices selected by the individual writer. 
Next in the article come ten lines of combat - one could call it agonistic or polemical - in which Names-Articles ("Libert (6)", "Reichlin (7)"), not persons, are called into question. The polemics of science obeys special rules; in this part, it consists in re-inserting modalizations into assertions (énoncés) that had claimed not to need them. The scientific assertion (l'énoncé scientifique), in fact, comes in two forms: the simple assertion (énoncé simple) and the modalized assertion (énoncé modalisé). It might be said that an assertion (affirmation) in the exact sciences is successful when it can appear naked, with no modalization, in the form "A is B." Failure is to remain stuck in modalizations ("Some claim that A is B"). ${ }^{10}$ The harshest attack imaginable is therefore to derail a given assertion and force it back into the conditions of its production. Thus: "L’un de nous a exprimé ses réserves sur les conclusions de Shibuzawa et coll" / One of us has expressed reservations about the conclusions of Shibuzawa et al. (p. 1,1.14); "N'ont pas été réunies toutes les conditions nécessaires pour affirmer que la fraction active de Schreiber et coll n'agit que..." / There were not present all the conditions necessary to confirm that the active fraction of Schreiber et al. acts only..., (p. 1,1. 19). Other Names-Articles are also summoned up to support the attack: "Reichlin (7) vient d'ailleurs de confirmer notre point de vue" / Reichlin (7), moreover, has recently confirmed our point of view (p. 1, 1. 15). Instead of simple assertions (énoncés simples) — "TRF exists", "A is B" — various types of modalisers are added, whether for an author - $X$ has said that " $A$ is $B$ " - for an argument - X's conclusions leading to the statement that " $A$ is $B$ " - or for a procedure - in vitro experiments that allow $X$ to conclude that " $A$ is $B$." It is often claimed that scientific ideology is a type of theatrical performance that hides what goes on in the wings and offers the audience a theoretical process with neither plot nor characters. In fact, closer observation of actual scientific activity shows that this is not the ideology of scientists, but rather that of philosophers who wish to impose it on scientists. The scientific operation par excellence is not to conceal the conditions of production but to put them in the place of the performance that authors wish to put on. The results may be disastrous for a hurried author who is trying to free himself as quickly as possible from all these conditions. It is even possible to define the frontiers of a science as the place where opponents are constantly forcing assertions (énoncés) back into the experimental conditions under which they were produced. Any "cold" science, in contrast, is presented as a sequence of affirmations - at least until a new front is established, remobilising assertions (mobiliser de nouveau les énoncés) and revealing their true origin." 


\section{The right to assert and its prerequisites}

The battle is fought over the right to assert (droit de dire) (that the active fraction is attributable to the substance TRF). What is at stake in this debate is therefore authority. They say they have isolated TRF but a close examination of their evidence reveals that they are not entitled to assert this. The Note, in total contrast, aims to convince the reader: before I might not have believed in the existence of the substance TRF; now I cannot but believe in it. Before I might have believed that Shibuzawa had isolated TRF; now I can no longer believe that. The transfer of authority is indissolubly professional and intellectual. Whoever is able, in the article, to accumulate sufficient authority to definitively convince others that he has indeed demonstrated the existence of the substance TRF wins the right to dominate the new field of study. The operation of convincing triggers recognition in the "souls" of one's peers. In other words, the author will receive credit. This credit - marked quantitatively by citations (cf. Chubin \& Moitra, 1975) - may, within the profession, be converted into a job and research funding, which when reinvested in the laboratory will make it possible to raise the stakes and take the substance farther. Although it would be premature to undertake a complete analysis of credit in the exact sciences, it can be seen that the right to assert (intellectual authority) entails the power to convince, which in turn, by means of the recognition it inspires, establishes a new (professional) right to assert (cf. Bourdieu 1976). These questions of entitlement are manifested at several points in our text by modalities which should not be present at all, if science were written in the way philosophers claim it is. For example, note "les réserves doivent s'appliquer" / certain reservations must apply (p.1,1.15) and "toutes les conditions nécessaires n'ont pas été réunies" / not all of the necessary conditions were assembled (p. 1,1. 19), which contrast with the phrases of line 7, "qui correspondent aux caractéristiques attendues" I which correspond to the expected characteristics and line 43 on page 2 , "qui correspondent aux caractéristiques hypothétiques attendues" / which correspond to the expected hypothetical characteristics. The entire text hinges on a point of entitlement; agreement or disagreement with this point confers or constitutes the authority to assert (l'autorité de dire).

Interestingly, the first author of the article had, in an earlier text, defined thirteen criteria that must be met in order to be able to conclude that a hypophysiotropic hormone had indeed been observed, and not an artifact. These thirteen criteria define the rules of the game, without which the preceding combat and the ensuing experimental edifice would be incomprehensible. A glance at this text from the Journal de physiologie ${ }^{* * *}$ suffices to show the omnipresence of ethical modalities (which express the obligation to proceed in certain ways), such as "il faut", "il faudrait" / one 
must, "on doit s'attendre" / it must be expected. Nonetheless, this is not a legal text in which right is based on a higher law, a natural law, or a precedent. Each requisite is derived directly from a long series of experimental failures (ten years spent on CRF without producing a clearly defined substance), considered by someone who, to quote Nietzsche, wishes neither to deceive nor to be deceived. If we were to seek a political equivalent to this manifest, we would find it in the notion, dear to Stephen Toulmin, of the "coup d'état", or even a settling of accounts with oneself or with others. In its 13 points, this text summarises the conditions of authority of the new field. If I wish to know, then I must perform the operations listed in 1 to 13 , in order to be able to assert (pouvoir dire) (that A is B). Authority is the means whereby the wish to know is translated into discipline in one's experiments. Rivals in the field of study do not really wish to know. Without great risk, they conclude from the paradigm that TRF must exist and that they actually see it. They live off the paradigm; they do not open up a new field. But if the desire to know is keen enough and if one wishes to go from TRF, a substance whose existence has been demonstrated (in the 1962 article), to TRF, a substance that is chemically identified (in the 1969 article), then one must be absolutely certain that the substance one has at the outset is TRF and not something else. The polemic against others is but the consequence of a polemic against oneself, and it is measured in the strength of one's desire to know. In denying oneself the authority to assert (ne s'autorisera pas à dire) that one has identified TRF before meeting the 13 criteria, the authority of others is undone. The game is worth the candle, for the result is the creation of a new field of study over which one has incontestable authority.

\section{The power to prove}

So far we have studied first sociological markers, then references, and lastly the strategy of shifts in the literature that the article achieves. Now we will examine what makes this strategy possible. Paragraphs 3, 4, and 5 (part B) seem so "technical" that they appear irreducible to any sociological analysis. Leaving aside for the moment paragraph 4 , which is simply a list of articles that have been published or are to appear, let us consider paragraph 5. If we superpose this paragraph on the list of 13 criteria, it becomes clear that each sentence comes from one of the criteria and goes toward part A of the text (paragraphs 1,2, and 6), thereby making it possible. This part of the text is therefore no more "technical" than the first part. The author does not give us his experimental protocols and logbooks - far from it. Instead, he argues, but he does so by means of other texts. It thus appear impossible to distinguish supposedly "hard" data from "soft" hypotheses because, from the first, the 
experimental procedure in its entirety has been a stratagem aimed at scoring a point in the battle to convince. Kudos to whoever can maintain, in an article of this kind, the philosophical difference between hypothesis and confirmation. If a difference is to be found, we must seek it either in military images - the difference between the front and the supply corps - or in gambling - the difference between a pile of chips that have been won and the bet that risks them all. To conclude from this that instruments are unnecessary and experiments are secondary is the admission that one has never fought a war and never gambled.

Paragraph 5 relates the tests to which hypothalamus extracts were put and which produced effects. These effects are almost always in the form of writing (diagrams, spectrograms, curves, photos) on writing surfaces. Paragraphs 3 and 4 describe on what kind of instruments and, in the bioassays, on what kind of "screen" the effects were recorded. Other articles describe exactly how the bioassays were done. Enormous effort was required to develop the screen upon which an image like that on page 4 could be drawn. A combination of manual dexterity, experimental creativity, and intelligence made it possible, for the first time, to capture a stable form on the instrument: this is the delayed peak on which the result of the TRF activity test is superimposed.

This is what explains the effect of paragraph 3 on a small group of readers. The number of hypothalimi is new and is clearly distinguished from the small quantities that had been used hitherto. The mention of pyridine and a "Sephadex G 25" column signals an innovative method. Similarly, in paragraph 4 the use of rats rather than mice is also a new element. The most important element for evaluating the credit of the preceding assertions (affirmations) is found here. In fact, every scientific account is preceded by an "instrumental" account - "instrumental" in the sense used in literary analysis - such as a description of the sword that will come into play in the hero's glorious struggle. This account defines the conditions for earning credit. In the present case, the argument is strengthened by this single technical paragraph. In reading it, one knows that these new methods will ultimately support the assertions (affirmations). Nonetheless, this instrument story is not our concern here, because in paragraph 5 it is considered completed.

Page 3 of the article provides details of the tests that the purified fractions undergo inside the instrument. At the end of each test there is a number. Obviously, these tables do not provide raw results and do not retrace the ins and outs of the actual experiments. Instead, they contain numbers that have been "cleaned up" and are ready to be used at any time in the argumentation. Let us consider anew this accumulation of numbers, so characteristic of the scientific text. Page 3 summarises a lengthy experimental protocol by mobilising a few numbers. Paragraph 5 mobilises 
the numbers of page 3 , but concludes with a modalization rather than a number. Paragraph 1 in turn uses the modalizations of paragraph 5, but its outcome, far from being a modalization, is a victory in the literature.

This accumulation of texts may be summarised graphically. The instrumental accounts cover the Note; the list of the 13 criteria defines the authority. In the middle, the textual layers of our article are accumulated. Upstream there are the other papers that are transformed by the text's operation; downstream there are those that transform it. This characteristic of accumulation explains why a laboratory is a type of production unit quite different from a factory. A pharmaceutical plant produces substances that produce money; a laboratory produces substances, which produce numbers, which give rise to modalizations, which convince. In the first case, substances are sold; in the second case, assertions (énoncés) earn credit. ${ }^{2}$ But the interplay between these two universal equivalents - money as capital and information - is in fact more complex.

\section{The authority of facts}

We have stated that paragraph 5 establishes modalizations - that is, it argues. The sentence "deux zones d'activité sont constamment retrouvées" / two zones of activity are found again and again is not a simple observation (constatation), but a strong modalization which enables the authors to state that they are dealing with a substance and not an artifact. This consistency, repeated on p. 2, 1. 29, is precisely what was lacking in other demonstrations. The fact that consistent effects are obtained "à partir de l'hypothalamus" / from the hypothalamus, while no effect is obtained on "l'extrait acétique du cortex cérébral" / acetic extract from the cerebral cortex is not reported for the mere pleasure of offering up a curious fact, but to prove that the experiment meets criterion 1 . With this sentence, a new objection is undone and the right to assert (droit de dire) is enforced. The test of table 2, on page 3 of the article, is recounted in line 25 of this paragraph. The experiment upon which it is based was devised from the beginning in order to meet criterion 3 , which requires that the stimulation follow a linear function of the logarithm of the dose injected. Once again, the agreement between the conditions imposed by the criterion and those obtained by the test scores a point for the assertion (affirmation) that TRF is present. We have used the term "épreuves" / tests to designate what might be called "expériences" / experiments. But the experiment - which took place in the laboratory, with animals, test tubes, and instruments - is not recounted here; that would take too long and would be pointless. Instead of experiments, paragraph 5 sets forth "tests," in the literary sense of the term. In the present case, these tests are complicated by the absence of 
the true prize at stake, which is actually to be found in the list of 13 criteria. Take line 10 , for example. We have two active fractionations. How can we tell which is the "right" one, and which is the "wrong" one? By putting them to a test, defined by criterion 6: in order to identify a hypophysiotropic hormone, activity must cease following a hypophysectomy. Not a word is said of the actual experiment as it took place in space and time, but the textual test is clear and can be summarised as follows:

\begin{tabular}{|l|r|r|}
\hline fraction A & hypophysectomy & activity \\
fraction B & no activity \\
\hline
\end{tabular}

The first and third columns define the actors; the middle column defines the action, or in this case the instrument. But it is clear that the test, if halted at this point, is devoid of meaning. It becomes meaningful only when a second test is added to the right, which is the test of convincing:

\begin{tabular}{|l|r|r|r|r|}
\hline fraction A & hypophysectomy & $\begin{array}{r}\text { activity } \\
\text { fraction B }\end{array}$ & requisite no. 6 & $\begin{array}{r}\text { can in no case be TRF } \\
\text { can definitely be TRF }\end{array}$ \\
\hline
\end{tabular}

It would be tedious to go over the entire paragraph in this fashion, but each sentence has the same organisation. Fraction B is judged to be different from TSH, in order to meet criterion 10; and then different from vasopressin, oxytocin, and long-acting TSH. At each instance, in the mind of the informed reader, another argument collapses, a hesitation is reinforced, an objection is undone. This paragraph is not "technical"; it is rather a discussion whose particular form is a threepronged reference to the tables on page 3 , the list of 13 criteria, and part $A$ of the text. In other words, it has a particular rhetorical shape.

The first sentence of paragraph 6 , which remains to be examined, amply confirms the perspective that we have adopted. This sentence summarises the convincing effects produced by each sentence in paragraph 5. "Les activités décrites plus haut (...) correspondent aux caractéristiques hypothétiques attendues de la neurohumeur TRF" / The activities described above ... correspond to the hypothetical characteristics expected of the neurosecretion $T R F$ (p. 2,1.43). The circle is closed; victory is achieved. The name TRF now designates a distinct object in the minds of readers and rivals. Eight years later, its content will change once again, and the name 
TRF will designate Pyroglu-His-Pro-NH2. The name of the main author (referring to both an individual and a group) is so firmly attached to the two changes in the signified entity that it achieves a sort of immortality.

However, the article dwells neither on the totals that allow this assertion to be credited nor on a crow of triumph. Instead it concludes with an operation of caution. On 1.40 of p. 2, we read that the activities are "attribuables à une ou plusieurs substances" / attributable to one or more substances. As soon as credit is obtained, it is assigned further limitations. The activity is attributable to a substance; nowhere is it claimed that it is the substance: that is the first act of caution. It is attributable to "one or more substances": the second act of caution. And lastly, in an admirable accumulation of modalizations, we read "sans pour autant préjuger que la substance TRF ainsi mise en évidence correspond certainement au médiateur physiologique" / without thereby prejudging that the substance TRF thus demonstrated definitely is the physiological mediator (p. 2, 1.44). There is no justification for squandering the undeniable credit earned by the Note by claiming that what has been proven in the laboratory is also true in the body. This is the third act of caution. Caution, as we have known since the time of the Greeks, is a strategy to protect oneself in advance against attack. In the present case, it is a discreet lesson in scientific comportment for those, such as Shibuzawa, who have been so rash as to give the chemical composition of a substance that had barely been isolated.

In this study we have performed two transformations: an extension and an inversion. We have extended to an article in the exact sciences an analysis whose concepts were developed for literary texts, and we have shown that scientific literature is a part of literature, thereby encouraging other researchers not to be intimidated by the apparent impenetrability of scientific texts. Nonetheless, this analysis cannot be extended to the scientific "genre" (if such exists), for our study deals with but a single article. The inversion, or reversal, we have performed is this: Science is always studied in speculative terms as an activity oriented toward nature. We have reversed this relationship, considering scientific production in more military terms, as a series of operations oriented towards the field of study, itself identified with frontier literature. In this perspective, nature provides the ammunition whereby offensive strikes are made invincible. We have introduced characters that were thought to be impossible on the theoretical stage: Stratagem, Right, Combat, Desire, and, most of all, Rhetoric. We have thus fulfilled one of the conditions of the sociology of science - to rediscover society within rationality, and, alone among all the sciences, disorder beneath order. As simplistic and limited as the above analysis may be, it opens three new research orientations. The first is directly literary: what constitutes a good or a bad scientific article? In his evaluations each researcher actualises a hitherto 
unawakened "competence," in the linguistic sense of the term, which, within the most quantitative sciences, defines countless degrees of quality. The second orientation is more properly philosophical: what constitutes a theory and how can an actual difference between rhetoric and demonstration be delineated? To the extent that one discovers polemic where one expected deduction, and production where one expected performance, the notion that philosophers have of science and of their own theoretical discourse must be modified substantially. The third orientation, which is directly sociological, poses the question of scientific credit. The economics of science examines how investment of capital increases production; the American model of the sociology of science studies how symbolic capital (authority) is accumulated by scientists; epistemology studies how argumentation is strengthened or weakened. The time has come to study science from the point of view of a general economy of credit, where credit is accreditation and capital - in the form of money, in the form of authority, and in the form of data. This article is intended as a preparatory step towards the study of the accumulation, circulation, and transformation of these various forms of credit.

\section{Postscript (Actes de la recherche)}

One of the authors of the article studied here had several objections to the analysis, which Bruno Latour was good enough to send us. They fall under three main headings:

\section{Its fetishistic approach:}

The article is but a means of transmitting information and has no importance in itself.

What ultimately underlies texts such as this is Nature.

Actual effects occur on other bodies, not on texts.

\section{Its blindness to the information transmitted and to the concreteness of the experiments:}

People will think that we filled up five pages saying what could have been stated in two lines. But in actual fact, our text is full of information. 
If details of the protocol are not given, it's not because we are trying to hide anything. It is simply unnecessary.

The results reported are not merely "sets of writings." It would be better to call them "symbols" or "languages." The results are merely translated by the instruments (...); we have no other means of obtaining these results, but the actual effects are independent. With all of the data together, we are able to construct a complete and coherent picture.

\section{Its naively cynical and polemical vision of scientific strategies and underestimation of epistemological obstacles:}

Everything is reduced to personal motivation, as if scientists were sneaky and conniving. We are conducting a study, not playing a game.

We discover, we do not create. From time to time, new relationships among ideas are suggested. We build things.

How long does it take to eradicate a misconception? How many years are necessary to demolish, step by step, a tiny but persistent mistake?

\section{Notes}

* The original French article is reproduced, with special permission from the editor and authors, on pages 87 to 106 of this Technostyle issue. Readers should be aware that Latour and Fabbri's article includes references to two other French texts to be found at the end of their article on pages 107 to 113 . [Note from the editors]

** This text is reproduced on pages 107 to 110 of this issue of Technostyle.

1 See the classic studies by D. de Solla Price (1965); cf. a recent application in C. Mullins . (1972).

2 With the notable exception of Gusfield (1976). However, this study deals with one of the "soft" sciences imitating the style it takes to be that of the "hard" sciences. Hence the author's conclusions, which are entirely different from ours.

3 We refer to the classic studies by Genette (1972), Benveniste (1970), and Greimas (1966).

4 See Greimas (1976).

5 A remarkable example of this phenomenon is given in Mulkay and Edge (1973). For a completely different perspective, see Foucault (1966).

6 See, in particular, Zuckermann (1968). 
7 It should be pointed out that the Comptes rendus publish only research notes and not articles. According to the author, these notes have no other purpose than "to intrigue the scientific audience so that it is almost certain it is convinced and will telephone to ask for the information which is missing in such a short report."

8 The article can be read as long as it is located at the frontiers of the discipline. Once it is integrated into cold science it no longer need be read, except to verify methodologies, to recreate the history of the discipline, or to evaluate the progress made. However, even if it is not read or reread, it can still be cited. The terms "used" or "taken apart" might be more accurate than "read." With the Science Citation Index, we can assess by whom this article has been used and for how long. The results are as follows: it has been cited 40 times since 1964 in articles produced outside the group and 14 times in articles by members of the group. Eleven articles confirm that this one was indeed the first to demonstrate TRF activity; this confirmation comes not only from founders of the paradigm but also from direct competitors. Seven articles confirm the text, but cite it along with texts by authors the text seeks to distinguish itself from. Among the five authors who cite the text for technical reasons, only one cites a detail that could be construed as information, the "débit de 50$60 \mathrm{ml} / \mathrm{h}$ " / rate of $50-60 \mathrm{ml} / \mathrm{h}$ ( page 1 , line 29 ).

9 For a recent presentation in French of the problem, see Schally and Kastin (1976). This article is interesting in that it was written by the group's competitor. It tells a different story, and even gives different names to substances.

10 The eponym (e.g. "the Compton effect") must not be confused with persistent modalizations. The former represents the height of prestige in the sciences, while the latter is a mark of criticism or derision. Thus, "Schally's GHRH did not prove to be active in our experiments" identifies a merely local substance that has no claim to be identified as GHRH. The genitive in this case is a mark of disdain.

11 Even years later it is possible to re-open protocol books and re-calculate the raw data. Despite the dominant perception, a scientific assertion is never free of its modalizations, unlike a work of art which eliminates its modalizations, and ideology, which covers its modalizations up.

*** This text is reproduced on pages 112 to 113 of this issue of Technostyle.

12 This remark, which is far too insufficiently nuanced, is intended merely to indicate a study to be undertaken.

\section{References}

Benveniste, E. (1970). Essais de linguistique générale (Vol. 1). Paris: Éditions du Seuil.

Bourdieu, P. (1976). Le champ scientifique. Actes de la recherche en sciences sociales, $2-3$.

Chubin, D. \& Moitra, S. (1975). Content analysis of references: adjunct or alternative to citation counting. Social studies of science, 5 (4) 423-41. 
Foucault, M. (1966). Les mots et les choses. Paris: Gallimard.

Genette, G. (1972). Figures III. Paris: Éditions du Seuil.

Greimas, A. (1976). Sémiotique et sciences humaines. Paris: Éditions du Seuil.

Gusfield, J. (1976). The literary rhetoric of science. American sociological review, 41 (1).

Latour, B., \& S. Woolgar. (1986). Laboratory life: the construction of scientific facts, second edition. Princeton, NJ: Princeton University Press.

Mulkay, M. J. \& Edge, D. O. (1973). Cognitive, technical and social factors in the emergence of radio astronomy. Information sur les sciences sociales, 12 (6), 2571.

Mullins, C. (1972). The development of a new scientific specialty. Minerva, 10.

Schally and Kastin (1976). Les hormones de l'hypothalamus. La recherche, 63.

de Solla Price, D. (1965). Little science-big science. New York: Columbia University Press.

Zuckermann, H. (1968). Patterns of name ordering among authors of scientific papers. American journal of sociology, 74 (3). 